FROM THE EDITORS' DESK

\title{
Screening for Intimate Partner Violence: The Time Is Now
}

\author{
Mitchell D. Feldman, MD, MPhil \\ Division of General Internal Medicine, Department of Medicine, University of California, San Francisco, San Francisco, CA, USA.
}

J Gen Intern Med 28(10):1251-2

DOI: $10.1007 / \mathrm{s} 11606-013-2576-0$

(c) Society of General Internal Medicine 2013

I $\mathrm{t}$ has been almost 20 years since the OJ Simpson trial first brought the issue of intimate partner violence (IPV) into the public eye. At that time, it was considered to be primarily a legal issue. Few practicing physicians had any knowledge of or interest in the subject, and the medical literature had only a small number of research articles on its detection and treatment. The first paper on what was then called 'domestic violence' appeared in JGIM in 1990. The title, "Domestic Violence and the Internist's Response: Advocacy or Apathy?" aptly summarized the medical establishment's stance. Unfortunately, apathy was far closer to the truth.

Over the past 20 years, research conducted in a variety of medical settings has underscored the high prevalence of IPV. It is estimated that about 4 million women are victims of intimate partner violence each year in the United States; about 500,000 of them required medical treatment. Women visiting outpatient medical and obstetric/gynecologic clinics as well as emergency departments (EDs) often do so for complaints directly attributable to IPV. Because they are frequently misdiagnosed, they may return time and time again, often with increasingly severe trauma. Cross-sectional studies from outpatient primary care clinics and EDs have found the prevalence of IPV among women to be as high as $30 \%$; lifetime prevalence rates up to $50 \%$ have been reported, and IPV accounts for more than half the murders of women in the United States every year. Many studies ask about violence exclusively in the context of heterosexual relationships; however, there appears to be a similar prevalence of IPV in gay, lesbian, bisexual or transgendered (GLBT) relationships, with the same physical and emotional consequences.

Experts and national groups such as the U.S. Preventive Services Task Force and the Joint Commission on Accreditation of Healthcare Organizations now advocate screening for IPV in health care settings, along with improved provider education. According to these experts, the potential benefits of screening outweigh the potential harms. Since research has demonstrated that most abused (and non-abused) patients favor routine screening by their health care practitioners, questions

Published online September 4, 2013 about IPV should be incorporated into the routine history and physical examination for all female patients. Some practitioners also screen men, particularly men in intimate relationships with other men.

In this issue of JGIM, Iverson et al. ${ }^{1}$ report on the accuracy of a brief IPV screening tool in a population of female Veterans Health Administration (VHA) patients. Participants completed a mailed survey consisting of the IPV reference "gold" standard [the 39-item Revised Conflict Tactics Scale (CTS-2)] compared with HITS, a brief screening tool consisting of four items that asks about physical hurts, insults, threats or screams over the prior 12 months. Responses to the HITS questions are assessed on a 5-point scale, with 1 indicating "never" and 5 indicating "frequently". The standard cutoff for a positive screen is 10 , but past studies have reported cutoff scores ranging from 6 to 11 . In this study, Iverson et al. found that $28.8 \%$ of their sample of female veterans reported IPV in the past 12 months. Compared with the CTS-2, the HITS performed reasonably well, detecting $78 \%$ of women identified as abused by the CTS-2 when a cut-off score of 6 was used. At this cut-off, they estimate a negative predictive value of $90 \%$ (i.e. that $90 \%$ of negative screens can be ruled out for IPV).

Several other papers in this issue of JGIM point to the importance of screening for what are otherwise often hidden problems in primary care. Ryan et al. ${ }^{2}$ report on a study that examined the prevalence of at-risk drinking in older adults with chronic medical conditions. They examined Medicare claims data for beneficiaries 65 years and older with one or more of seven common medical conditions, including dementia, diabetes and hypertension, and found that about one-third reported any alcohol consumption in a typical month, and about one in four of the drinkers (or about $7 \%$ ) reported at-risk drinking. For many of these older persons with chronic illness, any level of alcohol consumption may be problematic. For the one-fourth of drinkers who are consuming alcohol above guidelines, drinking may be a hidden cause of worsening morbidity and possibly death.

Also in this issue, Becker et al. ${ }^{3}$ discuss the challenge of appropriate opioid prescribing by primary care providers. They argue that to improve quality and reduce harms of opioid prescribing in primary care, well validated and brief screening tools are needed to measure safety, efficacy and 
potential misuse. Unlike for IPV and alcohol use, no such screening tools currently exist.

Of course, screening for IPV, problem drinking, opioid use and abuse, and other threats to our patient's health is just the first critical step that must also include appropriate treatment and/or referral by the primary care practitioner. While much progress has been made over the past 20 years in the recognition and treatment of IPV and other health problems that seem to sit outside of the traditional biomedical paradigm, much more needs to be done to reduce the burden of these often hidden but very real threats to our patients' health and well-being. As more and more primary care practices adopt electronic health records, the time has come to integrate screening tools for IPV, depression, substance use and other common medical and public health problems that are often unrecognized, but for which there exists evidence-based interventions that can enhance our patients' health, and perhaps even save their lives.

Corresponding Author: Mitchell D. Feldman, MD, MPhil; Division of General Internal Medicine, Department of Medicine, University of California, San Francisco, 1545 Divisadero, San Francisco, CA 94143-0320, USA (e-mail: mfeldman@medicine.ucsf.edu).

\section{REFERENCES}

1. Iverson KM, King MW, Resick PA, Gerber MR, Kimerling R, Vogt D. Clinical utility of an intimate partner violence screening tool for female VHA patients. J Gen Intern Med. doi:10.1007/s11606-013-2534-x.

2. Ryan M, Merrick EL, Hodgkin D, et al. Drinking patterns of older adults with chronic medical conditions. J Gen Intern Med. doi:10.1007/s11606013-2409-1.

3. Becker WC, Fraenkel L, Kerns RD, Fiellin DA. A Research Agenda for Enhancing Appropriate Opioid Prescribing in Primary Care. J Gen Intern Med. doi: 10.1007/s11606-013-2422-4. 